\title{
Lena Krauss \\ Maria, die Medea, die Bakchen und der Rhesos: Beispiele für die Euripides-Rezeption im Christus patiens ${ }^{1}$
}

\begin{abstract}
In this article, Lena Krauss discusses how the Christus patiens, the only extant dramatic cento from Antiquity, interacts with the three Euripidean plays $M e$ dea, Bacchae, and Rhesus. Most of the quotations of the Christus patiens belong to these three plays. Moreover, the Christus patiens can be divided into three parts based on the distribution of the quotation of these three plays over the Christus patiens. In the first part of the play, it is Mary who despairs because her son is dying and wishes to die herself. She does so in verses borrowed from the Medea. In the second part a different Mary enters the scene who reveals the Christian mystery mostly in verses borrowed from the Bacchae, and in the third part we find a Mary who welcomes the sun of the third day as a sign of her son's resurrection in verses borrowed mostly from the Rhesus. This article addresses the question of how the perception and possible interpretation of the character of Mary changes if and to the extent in which this intertextual play is taken into account. In the last section of the article the quasitransformation of Mary into a 'Mary-Medea' is addressed by placing the Christus patiens in the wider context of Christian drama.
\end{abstract}

Der Christus patiens ist ein christliches Cento-Drama in (bis auf wenige Ausnahmen) ${ }^{2}$ 2602 jambischen Trimetern ${ }^{3}$. Vorgeschaltet ist dem Drama, dessen Datierung bis heute umstritten ist, ${ }^{4}$ eine Präfatio, in welcher der Dichter ankündigt, vom „Welt-errettenden

1 Der vorliegende Artikel bietet einen Ausschnitt aus der noch unveröffentlichten Dissertationsschrift der Verfasserin zum Thema einer intertextuellen Lektüre des Christus patiens und seiner Marienfigur.

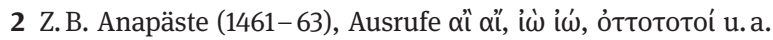

3 Bzw. in byzantinischen Zwölfsilbern (Hunger 1978, 106); zum byzantinischen Zwölfsilber siehe Hunger 1978, 91-93.

4 In jüngerer Zeit argumentiert Wojtylak-Heszen 2004 für die Autorschaft des Gregor von Nazianz (*329), Vakonakis 2011, 97-103 hingegen für eine Spätdatierung (11./12. Jh. n.Chr.). Der anhaltenden Diskussion um Autorschaft und Entstehungszeit des Christus patiens steht das Zeugnis der Handschriften gegenüber, von denen die meisten, darunter auch die wichtigen frühen, Gregor von Nazianz als seinen Autor nennen, wie Tuilier 1969 nachweist (Most 2008 stellt allerdings deren Zeugniswert in Frage). Zur Autor- und Datierungsfrage siehe ausführlich Trisoglio 1974 (mit einer chronologischen Darstellung der Arbeiten zur Autorfrage aus den Jahren 1571 bis 1971), Augusti 1816, Eichstädt 1816, Ellissen 1855, XX-LI, Brambs 1884, bes. 3-7, Tuilier 1969, 11-18, Swart 1990, 18-73 (mit einer thematischen Anordnung der Argumente für und gegen die Autorschaft Gregors aus Publikationen bis zum Jahre 1969 sowie einer chronologischen Anordnung der Publikationen zum Thema von 1969 bis 1990); Vakonakis 2011, 65-96.

https://doi.org/10.1515/9783110677072-021 


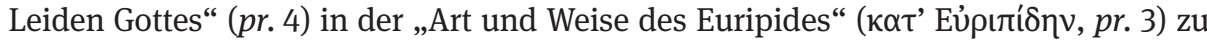
erzählen. Bei fast zwei Dritteln der Verse des Christus patiens handelt es sich um Zitate ganzer Verse oder Teilen davon vornehmlich aus den Tragödien des Euripides. Deswegen gilt der Christus patiens bis heute als das einzige aus der Antike erhaltene Cento-Drama.

Obwohl das Drama seit Bladus' editio princeps (Rom 1542) den Titel Xpıбтò

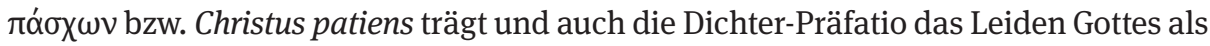
Thema des Dramas ankündigt, bestimmt nicht das Leiden Jesu Christi, sondern das seiner Mutter Maria fast die Hälfte der dramatischen Bühnenhandlung. ${ }^{5}$ Marias Trauer, ihre Wut und ihre Verzweiflung angesichts ihres am Kreuz sterbenden Sohnes sind nämlich nicht nur Thema des gesamten ersten und längsten Teils des Dramas (11133), sondern kommen vereinzelt auch noch in den beiden darauffolgenden Teilen vor (1134-1905 u. 1906-2531), bis Christus im dritten und letzten Teil seiner Mutter (u. a.) als der Auferstandene erscheint.

Basierend auf inhaltlichen Kriterien lässt sich der Christus patiens dreiteilen: in einen ersten Teil welcher die Verzweiflung Marias angesichts ihres leidenden Sohnes auf die Bühne bringt; in einen zweiten Teil, welcher neben der Kreuzabnahme und Beerdigung des Toten vor allem Marias und Johannes' große Offenbarungsreden enthält; und in einen dritten Teil, in welchem sich die Hoffnung aller im Drama erfüllt, wenn die vor dem Grab von Pilatus aufgestellte Wache, Maria, Maria Magdalena, die anderen Frauen, Johannes und Simon Petrus, die zwei Jünger auf dem Weg nach Emmaus sowie die Jünger im Hause der Mutter des Markus nacheinander Zeugen der Auferstehung des Toten werden. ${ }^{6}$

$\mathrm{Zu}$ einer der größten Auffälligkeiten des in vielerlei Hinsicht außergewöhnlichen Textes gehört, dass sich die Dreiteilung des Dramas nicht nur basierend auf inhaltlichen Kriterien durchführen lässt, sondern auch in Hinblick auf die intertextuellen Einschreibungen. Denn jeder der drei inhaltlich von einander unterscheidbaren Christus patiens-Teile - Kreuzigung Jesu und Marias Verzweiflung (Teil 1), Kreuzabnahme und Beerdigung Jesu sowie Marias und Johannes Offenbarungsreden (Teil 2), Erfüllung der Hoffnung Marias und anderer durch die Auferstehung Jesu Christi (Teil 3) - lässt sich einer bestimmten Euripides-Tragödie zuordnen, insofern als sich jeder dieser drei Christus patiens-Teile dominant in eine bestimmte Euripides-Tragödie intertextuell einschreibt, und zwar: Teil 1 (1-1133) in die Medea, Teil 2 (1134-1905) in die Bakchen und Teil 3 (1906-2531) in den Rhesos. Diese Auffälligkeit in der Häufig-

5 Dass Maria, die angesichts der Leiden ihres Sohnes selbst leidet, die tragische Handlung im Christus

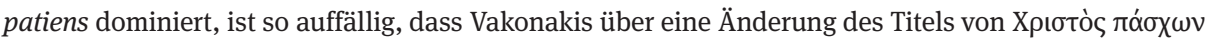

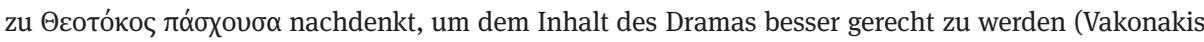
2011, 148).

6 Von einem christozentrischen Standpunkt aus überschreibt Tuilier 1969 den ersten Teil des Christus patiens mit „La Passion et la mort du Christ“ (9), den zweiten mit „Le Christ au tombeau“ (221) und den dritten mit „La Résurrection du Christ“ (283). Zu den Möglichkeiten der Gliederung des Christus patiens siehe ausführlich Tuiliers „Anaylse de la Pièce“ (19-26). 
keitsverteilung der drei Euripides-Tragödien Medea, Bakchen und Rhesos im Christus patiens soll Gegenstand der folgenden Untersuchung sein - und zwar in Hinblick auf die je spezifische Wirkung der sich daraus ergebenden intertextuellen Dialoge für den Christus patiens als christliches Drama.

Vorausgeschickt werden muss, dass diese Untersuchung aufgrund der Fülle der intertextuellen Verweisungen des Christus patiens hier nicht im Detail erfolgen kann. Den Ausgangspunkt der Einzeluntersuchungen bilden jeweils die ersten intertextuellen Einschreibungen des Christus patiens in die Medea, die Bakchen und den Rhesos, die von Einzeluntersuchungen weiterer, folgender intertextueller Einschreibungen des Christus patiens in diese Tragödien begleitet werden. Ziel ist es, ihre intertextuellen Dialoge mit Blick auf ihre bedeutungstragende Wirkung für den Christus patiens als christliches Drama insgesamt sukzessive in einem (imaginären) intertextuellen Lektüreprozess aufzuzeigen. ${ }^{7}$ Am Schluss des hier zur Diskussion gestellten intertextuellen Lektüreprozesses steht eine Aussage über die generelle, bedeutungsrelevante Wirkungsweise der intertextuellen Dialoge des Christus patiens mit der Medea, den Bakchen und dem Rhesos und damit auch eine Aussage über die generelle Art und Weise ihrer jeweiligen, spezifischen Rezeption im Christus patiens. Fraglos handelt es sich bei der hier vorgeschlagenen Wirkungsweise um nur eine von weiteren, möglichen und von anderen Lesern ebenso konkretisierbaren Wikungsweisen. Diese müssen sich allerdings jeweils an der Plausibilität einer intertextuellen Lesart des Christus patiens in Hinblick auf seine Gesamtinterpretation als eines chritlichen Dramas messen lassen.

Im Folgenden werden daher in drei Abschnitten die intertextuellen Verwendungsweisen und die darauf basierenden (möglichen) bedeutungstragenden Wirkungsweisen der intertextuellen Dialoge des Christus patiens mit der Medea, den Bakchen und dem Rhesos herausgearbeitet, jeweils beginnend mit den ersten intertextuellen Einschreibungen des Christus patiens in diese Euripides-Tragödien. Im vierten und letzten Abschnitt dieser Darstellung wird schließlich auf Grundlage dieser in den vorangehenden Abschnitten herausgearbeiteten möglichen Wirkungsweisen neben der Zusammenfassung auch eine Erklärung dafür zur Diskussion gestellt, warum mit dem ersten und längsten Teil des Christus patiens eine Marienfigur im

7 Dahinter steht die Annahme, dass die intertextuellen Einschreibungen des Christus patiens in eine Euripides-Tragödie von einem Leser während seiner intertextuellen Lektüre sukzessive mit einer spezifischen Wirkungsfunktion belegt werden. Dies geschieht, indem der Leser jeweils Mutmaßungen über die Deutung eines in Frage stehenden intertextuellen Dialogs des Christus patiens mit einer Euripides-Tragödie und über dessen bedeutungsrelevante Wirkung für den Christus patiens insgesamt anstellt. Dabei misst er jede seiner diesbezüglichen Mutmaßungen an seinen vorangehenden Mutmaßungen. Er liest also den jeweils in Frage stehenden intertextuellen Dialog und seine bedeutungsrelevante Wirkung für den Christus patiens insgesamt im Lichte vorangehender Mutmaßungen und passt dabei entweder seine momentane Mutmaßung dort hinein oder aber korrigiert die vorherigen Mutmaßungen im Sinne der jeweils in Frage stehenden Deutung. 
Zentrum des Dramas zu stehen scheint, die durch die maßgeblichen intertextuellen Verweisungen auf die Medea gewissermaßen als eine , Maria-Medea' konstruiert ist.

\section{Zur intertextuellen Verwendungs- und Wirkungsweise der Medea im Christus patiens}

Der erste und längste Teil des Christus patiens (1-1133), in welchem Maria immer wieder angesichts der Leiden ihres Sohnes dermaßen zu verzweifeln droht, dass sie ihrem Leben am liebsten sofort ein Ende bereiten würde, beginnt mit einer intertextuellen Einschreibung in die Medea und endet auch mit einer. Auffällig ist hierbei nicht nur, dass der erste Vers des Christus patiens sich in den ersten Vers der Medea intertextuell einschreibt, sondern auch, dass es sich bei den letzten vier Versen dieses ersten Dramenteils um Zitate der letzten vier Verse der Medea handelt. Dazwischen befindet sich - angefangen beim Dramenprolog (1-90) und seinen zahlreichen intertextuellen Einschreibungen in den Ammen-Prolog aus der Medea (1-49) - eine solche Vielzahl an intertextuellen Einschreibungen in so unterschiedliche MedeaSzenen, dass man sagen kann, im gesamten ersten Christus patiens-Teil werde die gesamte Medea vom ersten bis zum letzten Vers intertextuell aufgerufen.

Den Beginn des intertextuellen Dialogs des Christus patiens mit der Medea stellt der Dramenprolog dar. Hier wird Evas Fehler, im Paradies einen Apfel vom Baum der Erkenntnis genommen zu haben, im intertextuellen Dialog mit dem Ammenprolog aus der Medea als quasi-erotisches Verlangen nach dem Apfel gedeutet. Denn wie Medea

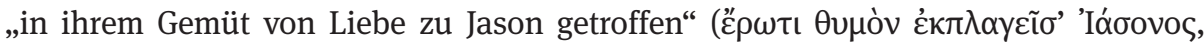
Med. 8), so begeht auch Eva im Paradies, „in ihrem Gemüt von Liebe zur Frucht ge-

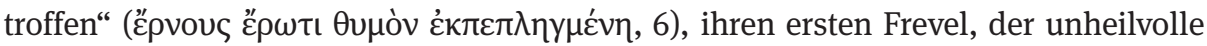
Folgen für sie selbst und ihre Kinder, nämlich die ganze Menschheit, ${ }^{8}$ mit sich bringt. Infolge dieses intertextuellen Dialogs also steht Eva wie Medea unter dem unheilvollen Einfluss des Eros, der sie nach der Frucht greifen lässt, verrät wie Medea den Vater (Gott : Aietes), verlässt wie diese die Heimat (Paradies : Kolchis) und kommt wie diese in eine ihr und ihren Kindern feindlich gesonnene, neue Heimat (die Erde : Korinth), in welcher diese schließlich unweigerlich den Tod finden (die Sterblichkeit der Menschheit als Kinder Evas : Medeas Tötung ihrer Kinder in Korinth).

Das Unglück der Menschheit, mit welchem im Christus patiens die dramatische Handlung beginnt, nimmt daher bei einer intertextuellen Lektüre des Dramenprologs in dem quasi-erotischen Begehren ${ }^{9}$,Eva-Medeas ‘ für den Apfel seinen Anfang und hat

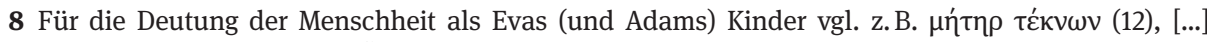

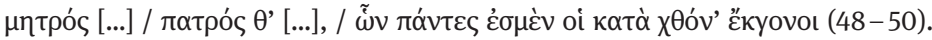

9 Im Zusammenhang der hier vorgeschlagenen Lesart eines erotisch bzw. quasi-erotisch motivierten Sündenfalls sei an die Vielzahl eindeutig erotischer Exegesen des Genesis-Geschehens insbesondere ab dem 1. Jh. n.Chr. verwiesen, welche aus Gen 3 „eine Geschichte voll körperlicher Sinnlichkeit“ 
den Tod von Evas Kindern (= der Menschheit) auf feindlicher Erde zur Folge. Dabei lässt Eva und Medea insbesondere ihre Handlungsmotivation als miteinander vergleichbar erscheinen: Beide Frauen begehen ihren Verrat an Vater und Heimat in einem erotischen bzw. quasi-erotischen Begehren, welches sie bestehendes Recht und bestehende Ordnung über Bord werfen lässt. Beide Frauen begehen in dieser Lesart also in erotischer Raserei einen Fehler, den sie bei klarem Verstand nicht begangen hätten.

Eine weitere Möglichkeit für den Leser, die Wirkung dieses intertextuellen Dialogs für den Christus patiens zu konkretisieren, besteht darin, sein Wissen um die überaus zahlreichen Zeugnisse aus der Antike für einen Eros zu aktivieren, der im Menschen durch die von ihm ausgelöste Raserei den Verstand löst. ${ }^{10}$ Denn spätestens seit Apollonios' Argonautenepos ist die Medeafigur zum Inbegriff für jemanden geworden, der von Eros seiner klaren Urteilskraft beraubt wird und eigentlich Unwagbares wagt. Apollonios' berühmte Verführungs- und Entscheidungsszenen im dritten Buch der Argonautika beschreiben, reich an Bildern, die komplexen Wirkungen des erotischen Pfeils im Herzen Medeas, aus deren „Brust“ sich hier neben anderem auch der „klare Verstand verflüchtigt“. ${ }^{11}$ Durch den intertextuellen Dialog des Dramenprologs und seiner Eva-Figur mit Euripides' Medea und der literarischen Tradition der Medea-Figur kann also die Motivation dafür nachgereicht werden, warum Eva vom Apfel kostet, nämlich weil sie bei seinem Anblick in solch eine ,erotische“ und damit auch verstandeslösende Raserei verfällt, dass sie dem Gebot Gottes keine Folge mehr leistet bzw. leisten kann - ganz wie Medea, die, beim Anblick Jasons von Liebe zu ihm getroffen, nicht mehr an das Verbot ihres Vaters denkt. Der Verstand, mit dem beide Frauen den Geboten ihres Vaters Folge leisten müssten, ist von Eros besiegt.

machen, um in der „Sinnlichkeit und Sexualität ..., verbunden mit der Frau, den Anfang der Sünde festzumachen“ (Küchler 1986, 48): z. B. slHen 31,6; 4Makk 18,7-9a; ProtEvJak 13,1; bSota 9b; Targum PsJonathan 4,1 (vgl. 5,3); bJebamot 103b (= bAboda Zara 22b); ApkAbr 23,1-8.

10 Vgl. die Diskussionen über den von Eros Getroffenen als jemanden, der den Verstand verloren hat und rast, z. B.: Plat. Rep. 3, 403a; Arist. Eth. Nic. 7,3, 1147a10-19, 1152b, Eth. Eud. 3, 1229a; Gorg. DK 82 B 11,19; Chrysipp. bei Diog. Laert. 7,113, Epik. bei Diog. Laert. 10,118. Oder vgl. die Dichter, bei welchen Eros den „Menschen den Sinn in der Brust und besonnen planendes Denken [bezwingt]“ (Hes. Theog.

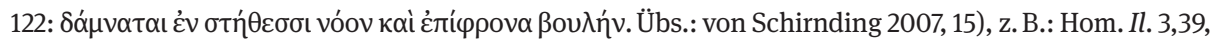
Od. 4,261-264; Pind. P. 2,26; 3,24; Sappho fr. 16,6-14 (PLF), 359 (PMG); Archil. fr. 191 (IEG); Anakr. fr. 359, 398, 83 (PMG); Aesch. Suppl. 108-111; Theokr. Eid. 2,135-137. Siehe zu diesem Thema ausführlich Thornton 1997, bes. 17: „Erotic passion is fundamentally a form of insanity, a destruction of the rational mind's control over the body, a suspension of appetites and emotions“ (Hervorhebung im Original).

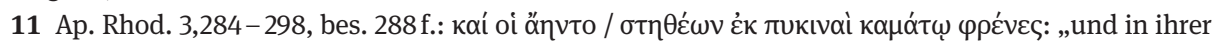
Verwirrung verflüchtigte sich aus ihrer Brust der klare Verstand“ (Übs. Glei/Natzel-Glei 1996, II,17). Medea wird zwar die für sie, ihre Familie und ihre Heimat unheilvollen Folgen ihrer Liebe zu Jason klar erkennen (vgl. z. B. Medeas Entscheidungsmonolog bei Ap. Rhod. 3,760-824), ihrem erotischen Begehren aber - gegen ihren Verstand, der erkannt hat, welches Unrecht sie zu tun bereit ist - nicht widerstehen können. 
Maria nun verleiht ihrem Schmerz, an dem sie wegen der Leiden ihres Sohnes vor allem im ersten Teil des Christus patiens immer wieder in Medea-Versen gänzlich zu verzweifeln droht, erstmals im Dramenprolog Ausdruck. Eine weitere Wirkung, die sich aus der intertextuellen Lesart des ,erotischen' Paradiesgeschehens ergibt, besteht nun darin, dass auch Marias Verzweiflung zum quasi-erotischen Wahnsinn wird, von welchem sie wie Eva und Medea getroffen wird, wenn sie das Leiden ihres Sohnes sinnlich wahrnimmt wie Eva die Frucht und Medea Jason. Maria spricht nämlich im Dramenprolog nur wenig später, nachdem sie Evas Eros nach dem Apfel und den daraus resultierenden Sündenfall geschildert hat, vom Schwert, das ihr, wie Simeon einst prophezeit hatte, ${ }^{12}$ beim Anblick der Leiden ihres Sohnes durch die Seele dringen werde. Den Schmerz, den ihr dieses Schwert bereite, beschreibt Maria dabei in Bildern, die vor dem Hintergrund der intertextuell-,erotischen' Lesart des Paradiesgeschehen zum Pfeil des Eros werden können, der in ihrem Herzen wirbelt. Marias Verzweiflung also, in die sie aufgrund der sinnlichen Wahrnehmung ${ }^{13}$ der Leiden ihres Sohnes gerät, können damit zum ersten Mal im Christus patiens im Dramenprolog zum Ausdruck einer quasi-erotischen Raserei werden, in welcher Maria, weil diese ihr den Verstand löst, nicht mehr Herrin über ihre Sinne ist:

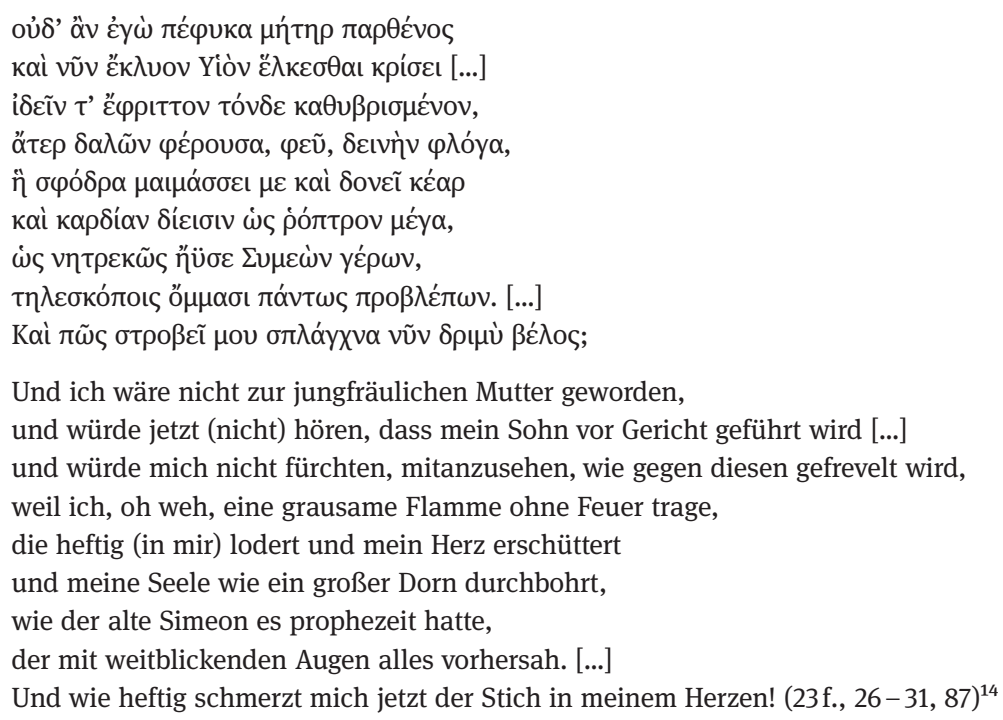

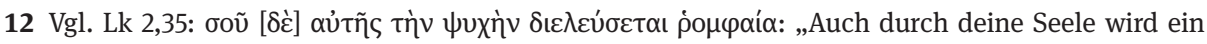
Schwert dringen“ (Übs. Lutherbibel).

13 Im Dramenprolog hat Maria vom Leiden ihres Sohnes zwar bisher nur gehört (ع̋k $\lambda v o v, 24)$, fürchtet sich aber explizit vor dessen Anblick (26), und später, als sie ihren Sohn in seinem Leiden mit eigenen Augen sieht, verzweifelt sie daran (siehe hierfür z.B. die auf S. 406 zitierte Marienrede mehrheitlich in Medea-Versen).

14 Sofern nicht anders vermerkt, stammen alle Übersetzungen von der Verfasserin. 
Es fällt auf, wie wenig der Dichter Marias Beschreibung ihres Schmerzes nach Simeon, den sie im Prolog namentlich aufruft (30), sprachlich an Lk 2,35 anschließt. Es ist im

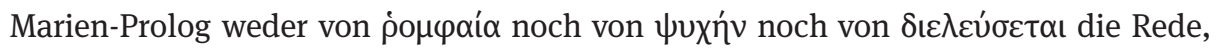

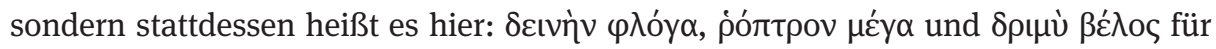

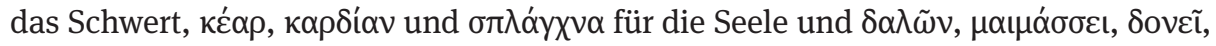

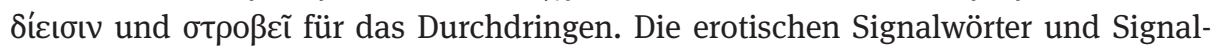
bilder, derer sich die verzweifelnde Maria sowohl hier als auch im weiteren Verlauf des Dramas immer wieder bedient, sind zahlreich und lassen sich nicht nur an die berühmte Verführungsszene bei Apollonios Rhodios anschließen, sondern auch an die reiche griechische Tradition von Bildern, derer sich griechische Dichter und Denker bedienen, wenn sie über die Auswirkungen der (erotischen) Liebe auf Körper und Seele sprechen. So beschreibt die Maria im Christus patiens den Zustand, in den sie angesichts der Leiden ihres Sohnes körperlich wie seelisch gerät, u.a. als Sprachlosigkeit, ${ }^{15}$ als schmerzhaftes Getroffen-Sein von Stichen und Pfeilen im Herzen, ${ }^{16}$ als unerträgliches Brennen im Innern, ${ }^{17}$ als Raub des klaren Verstandes und Unfähigkeit, gemäß dem eigentlich verstandesgemäß Erkannten zu handeln, ${ }^{18} \mathrm{~d}$. h. hier konkret: als Unfähigkeit, über das Leiden ihres Sohnes nicht zu verzweifeln, sondern sich darüber zu freuen, weil sich damit der göttliche Heilsplan zur Rettung der Menschheit endlich erfüllt, wie sie selbst wiederholt an anderen Stellen im Drama sehr wohl versteht. ${ }^{19}$

Immer wieder bedient sich Maria in ihren Verzweiflungsreden, welche den ersten Teil des Christus patiens dominieren, der Sprache aus der Medea, durch die vor ihr Eva als erotisch Rasende wie Medea lesbar geworden war. Nicht nur ergibt sich durch die stete Unterfütterung der verzweifelnden Maria mit Medea-Versen im intertextuellen Kommentar eine ,Maria-Medea', sondern diese wird dadurch auch an die ,Eva-Medea' aus dem Dramenprolog angebunden. Denn auf intertextueller Ebene ist damit etwas in Maria, nämlich eine Art Medea-Element, insofern als sich beide Frauen im Christus patiens derselben vorgeprägten Sprache aus der Medea bedienen. Dieses, MedeaElement‘ konnte im Falle der ,Eva-Medea' erklärt werden als Entscheidung, die in einem vom Anblick der Frucht ausgelösten, quasi-erotischen Wahnsinn gegen den Verstand getroffen worden ist, und das scheint nun auch auf die ,Maria-Medea zuzutreffen. Denn das ganze Drama hindurch wird Marias Verzweiflung sowohl von ihr

15 Sprachlosigkeit: z. B. 720. Vgl. Ap. Rhod. 3,283; Sappho fr. 31,7-9 (PLF).

16 Stiche: z. B. 29, 356, 749.Vgl. Eur. Ba.795; Ap. Rhod. 3,275; Aesch. Suppl.109f.; Eur. IA 547; Eur. Hipp. 39 u. 1300; Plat. Rep. 9, 573a. Für Schmerzen durch Eros allgemein vgl. Ap. Rhod. 3,761-775; Sappho fr. 1,3f. u. fr. 31 (PLF); Theocr. Eid. 2,82-90 u. 106-110 (siehe ausführlich bei Thornton 1997, 34 u. 42). Für Pfeile vgl. Ap. Rhod. 3,286 u. 279; Aesch. Supp. 1004f.; Eur. Med. 632-634; Theocr. Eid. 23,5 (siehe ausführlich bei Thornton 1997, $40 \mathrm{f}$.).

17 Feuer: z.B. 27. Vgl. Ap. Rhod. 3,287, 296f.; Aesch. Prom. 649f.; Sappho fr. 31,9 (PLF); Theokr. Eid. 2,82f. (siehe ausführlich bei Thornton 1997, 32).

18 Z.B. 590 - 597, 605-609, 720 - 724, 743f., 745-750, 767-770, 874-877, 891-900, 1008-1011.Vgl. die in Anm. 11 genannten Textstellen.

19 Siehe hierfür z.B. die auf S. 406 zitierte Marienrede in mehrheitlich Bakchen-Versen. 
selbst als gegen ihren Verstand gerichtete emotionale Reaktion bezeichnet ${ }^{20}$ als auch von allen anderen Dramenfiguren als verstand- und maßlos kritisiert. ${ }^{21}$

$\mathrm{Zu}$ den Euripides-Versen, die im Christus patiens am häufigsten rezipiert werden, scheinen daher nicht zufällig jene Medea-Verse zu gehören, in denen Medea kurz vor dem Kindesmord ihre Unfähigkeit benennt, die unheilvolle Führerschaft ihres Be-

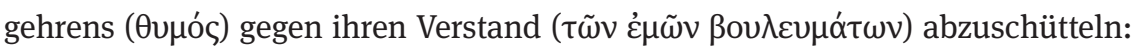

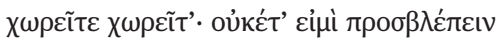

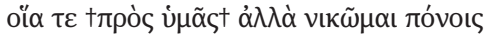

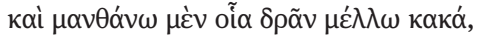

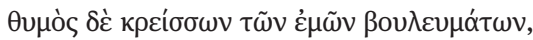

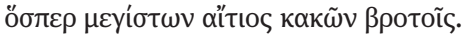 \\ Fort, fort, nicht länger ertrage ich, euch (= die Kinder) anzusehen! \\ Ich weiß, welche furchtbare Tat ich zu tun im Begriffe bin. \\ Doch stärker als mein klarer Verstand ist mein Begehren, \\ welches den Menschen schuld am größten Unheil ist. (Eur. Med. 1076-1080)
}

Wiederholt betont auch Maria im Christus patiens unter eindeutiger intertextueller Bezugnahme zu eben jenen Medea-Versen ihre Unfähigkeit, ihr ,Begehren' nach Tränen, d.h. ihre Verzweiflung über den Tod ihres Sohnes, abzuschütteln, obwohl sie es doch ganz ähnlich wie Medea eigentlich besser weiß:

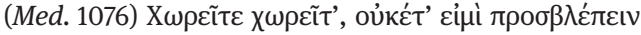

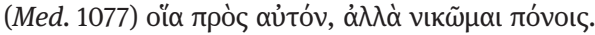

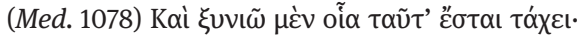

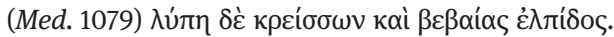

Fort, fort, nicht länger ertrage ich, meinen Blick

auf ihn zu richten, sondern ich werde überwältigt von Schmerzen.

Zwar verstehe ich, was bald (schon) sein wird,

doch (mein) Kummer ist stärker als (meine eigentlich so) sichere Hoffnung (auf Erfüllung des göttlichen Heilsplanes). (874-877) 22

Im intertextuellen Dialog dieser Christus patiens-Verse mit der Medea wird auf Medeas Unfähigkeit verwiesen, sich gegen ihr Begehren nach Rache an Jason durchzusetzen. Diese Unfähigkeit Medeas in Korinth wiederum korrespondiert mit Medeas Unfähig-

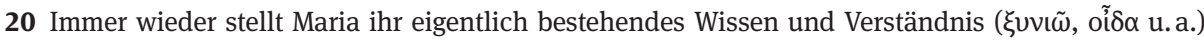

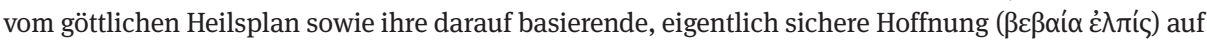
die Erfüllung dieses Planes in Opposition zu ihren tränenreichen Reaktionen unter dem Kreuz: $\mu \varepsilon^{v}$ $\delta \varepsilon ́$. Für Stellenangaben siehe das Zitat 874-877 auf dieser Seite sowie die dazu gehörigen Stellenangaben in Anm. 22.

21 Z. B. 617-620 (Marias Freundinnen, der Chor); 730 - 737 (Marias Sohn, Jesus); 1163-1165, 12411243, 1266 - 1268 u. 1286f. (Joseph von Arimathia); 932; 962; 970 - 982 (Johannes).

22 Vgl. 590 - 597 (mit Med.1077-1079); 720 - 724 (mit Med.1078f.); 743f. (mit Med. 1079); 767-770 (mit Med. 1079). 
keit in Kolchis, wo sie, von Liebe zu Jason getroffen, diesem Begehren nicht zu widerstehen vermag und die für sie und andere unheilvolle Entscheidung trifft, Vater und Heimat zu verlassen. Die Medea der gleichnamigen Tragödie erscheint damit insgesamt als jemand, der seinem momentanen Begehren trotz besseren Wissens Einhalt zu gebieten unfähig ist. Und eben weil dies so ist, und weil der erste erotisch motivierte Fehler Medeas im Marien-Prolog als Folie für Evas Frevel im Paradies lesbar geworden ist, erscheint nun Maria in den eben zitierten Versen, welche Medeas zweite Raserei in Korinth (d.i. ihr zweites gegen den klaren Verstand gerichtetes Begehren nach Rache an Jason) aufrufen, in ihrer Raserei (d.i. Marias gegen den klaren Verstand gerichtetes Begehren nach Tränen) wie Medea - und damit zugleich auch wie Eva, die im Prolog des Christus patiens durch Medeas erste Raserei in Kolchis (d.i. Medeas erstes gegen den klaren Verstand gerichtetes Begehren nach Jason) lesbar geworden war. Die Maria des Christus patiens, welche im weiteren Dramenverlauf wiederholt in Medea-Versen verzweifelt und ihre Verzweiflung in den eben zitierten Worten der Medea begründet, welche unfähig ist, auf ihren Verstand zu hören, erscheint daher nach der ,Eva-Medea' als ,Maria-Medea'. Das erotische Fehlgehen Medeas und ihr erotischer Wahnsinn, den sie weder in Kolchis noch in Korinth abzuschütteln vermag, wird in dieser Lesart zu dem verbindenden Element zwischen der Eva(-Medea) und der Maria(-Medea) im Christus patiens und das, wofür die Medea besonders in dessen erstem Teil rezipiert zu werden scheint.

Wie ,Eva-Medea‘ im Paradies dem Anblick des Apfels (wie Medea dem Anblick Jasons) nicht widerstehen kann, so kann sich in dieser Lesart auch ,Maria-Medea dem tränenauslösenden Anblick des leidenden Sohnes (wie Medea dem Anblick ihrer Kinder) nicht entziehen. Wie Medea kurz vor der Ermordung der Kinder, so gerät also auch Maria beim Anblick ihres Kindes in einen Widerstreit zwischen Verstehen und Begehren: Medea versteht, dass die Ermordung der Kinder ihr selbst den größten Schaden bringt, begehrt aber die Rache an Jason mehr; Maria versteht, dass ihr Sohn als Sohn Gottes sterben und wiederauferstehen wird, begehrt aber beim Anblick der konkreten Leiden ihres Sohnes, nur noch zu weinen.

Durch den intertextuellen Dialog mit der Medea lässt sich also Marias Schmerz als ihr quasi-erotisches Getroffen-Sein vom Wahrnehmungseindruck des leidenden Sohnes erklären - wie Eva im Paradies quasi-erotisch vom Wahrnehmungseindruck des Apfels und Medea in Kolchis erotisch vom Wahrnehmungseindruck Jasons getroffen worden sind. Erotischen Pfeilen gleich dringt der Wahrnehmungseindruck des leidenden Sohnes in Marias Seele ein und löst ein quasi-erotisches Begehren zu weinen in ihr aus. Dabei „verflüchtigt“ (Ap. Rhod. 3,288f.) sich ihr Verstand, mit dem sie eigentlich das Leiden ihres Sohnes als Teil des göttlichen Heilsplans verstehen könnte, wie sie es an anderen Stellen im Drama eindrücklich demonstriert.

In Zusammenhang mit dieser Lesart fällt auf, dass mit Marias Verzweiflung im Christus patiens immer ihre sinnliche Wahrnehmung der Leiden ihres Sohnes einhergeht, und zwar immer unter prominenter intertextueller Einschreibung in die Medea, wie etwa an der folgenden Stelle: 


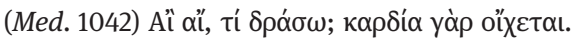

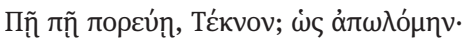

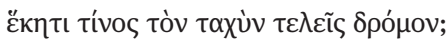

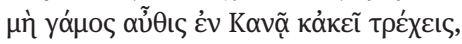

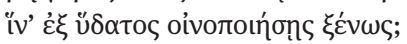

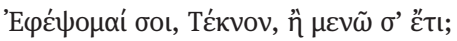

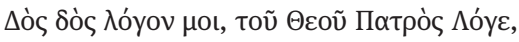

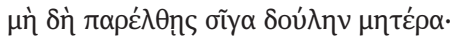

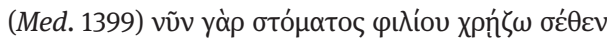

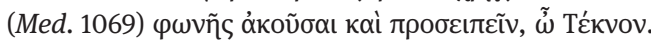

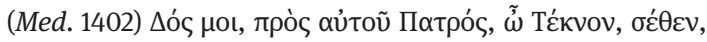

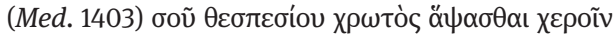

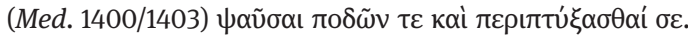

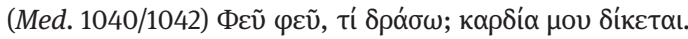

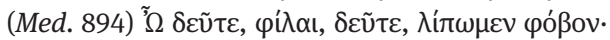

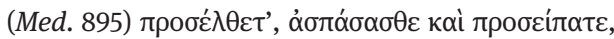

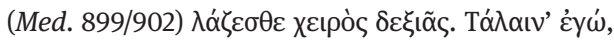

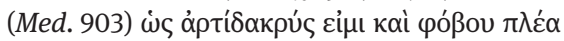

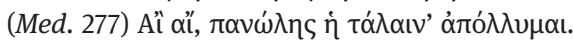

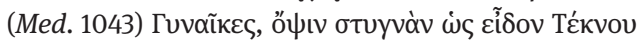

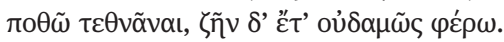

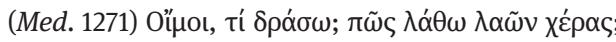

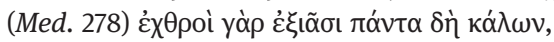

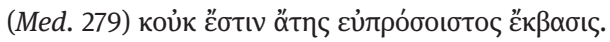

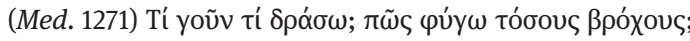

Ach, was soll ich tun? Mein Mut ist dahin.

Wohin, wohin setzt du deinen Fuß, mein Kind? Wie bin ich verloren!

$\mathrm{Zu}$ welchem Ziel wünschst du so schnell zu laufen?

Es ist (wohl) nicht wieder die Hochzeit in Kanaa, zu der du läufst, um dort auf wundersame Weise aus Wasser Wein zu machen?

Darf ich dir folgen, mein Kind, oder soll ich hier auf dich warten?

Gib, gib mir ein Zeichen, du Logos des göttlichen Vaters,

geh nicht schweigend an deiner Dienerin, deiner Mutter vorüber.

Ich möchte so gern aus deinem lieben Mund

deine Stimme hören und mit dir sprechen, mein Kind.

Gestatte mir, mein Kind, bei deinem Vater,

deinen göttlichen Leib mit meinen Händen zu berühren

und deine Füße anzufassen und dich zu umarmen.

Ach weh, was soll ich tun? Mein Mut schwindet.

Oh hierher, Freundinnen, wir wollen von unserer Furcht ablassen.

Kommt zu mir, umarmt mich und sprecht mit mir,

fasst (mich) an der rechten Hand. Ich Unglückselige:

Ich bin kurz davor zu weinen, bin übervoll von Furcht.

Ach, ich Unglückliche bin am Boden zerstört, ich gehe zugrunde.

Ihr Frauen, jetzt, wo ich den umnachteten Blick meines Kindes gesehen habe,

will ich sterben. $\mathrm{Zu}$ leben ertrage ich nicht mehr.

Ach weh, was soll ich tun? Wie soll ich mich vor den Händen des Volkes verstecken?

Denn die Feinde sind gegen alles Schöne ausgelaufen,

und es gibt keinen gangbaren Ausweg aus dem Unglück.

Was also soll ich tun? Wie so vielen Stricken entkommen? (453-477) 
In dieser Rede blickt Maria zum ersten Mal im Drama auf ihr leidendes Kind und wird von seinem Anblick schmerzhaft und offenbar auch verstandeslösend getroffen: Sie

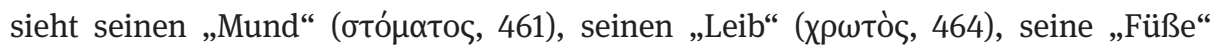

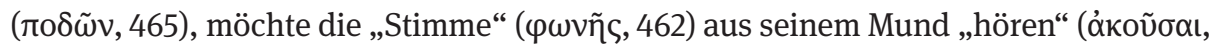

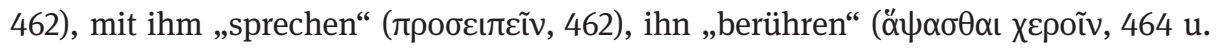

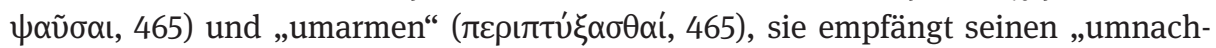
teten Blick“ (őభıv oтuyvòv, 472) und gerät in Panik. Ihre Sprache ist geprägt von hastig ausgestossenen rhetorischen Fragen (453-458), kurzen Kola und Schmerzensschreien

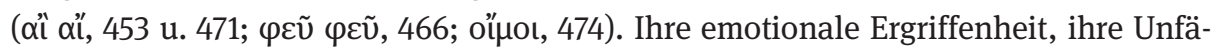
higkeit, klare Gedanken $\mathrm{zu}$ fassen und $\mathrm{zu}$ formulieren sowie das momentane Geschehen von seinem Ziel her angemessen(er) zu verstehen, ist deutlich hörbar. Sie steht ganz unter dem Eindruck der Leiden ihres Sohnes, unfähig, das momentane Geschehen innerhalb des göttlichen Heilsplans als etwas anderes zu sehen, als was es ihr gerade zu sein scheint. Die Fülle der körperlich-sinnlichen Details in Marias Rede machen dabei deutlich, wie sehr Maria hier unter dem Einfluss des sinnlich Wahrnehmbaren steht, das unheilvoll auf sie eindringt. ${ }^{23}$ Immer in Medea-Versen bekommt Maria dabei das momentane Geschehen nicht mehr mit dem göttlichen Heilsplan zusammen, ${ }^{24}$ versteht das Ziel des Sterbens ihres Sohnes nicht mehr ${ }^{25}$ und begehrt in äußerster Verzweiflung, lieber selbst zu sterben. ${ }^{26}$

$\mathrm{Zu}$ solch einer Maria, die sich im Christus patiens nur schwer dem sinnlichen Wahrnehmungseindruck vom leidenden Sohn entziehen kann und diesem insbesondere im ersten Dramenteil wiederholt verständnislos gegenübersteht, passt es, dass sie ihre maßlose ${ }^{27}$ Verzweiflung erst dann beendet, nachdem ihr der sinnliche Wahrnehmungseindruck vom sogenannten Doppelquellwunder ${ }^{28}$ das Gott-Sein ihres Sohnes am Ende des ersten Dramenteils unmissverständlich wieder vor Augen gestellt hat. Dass Maria sich infolge des Doppelquellwunders von ihrer Verzweiflung am Ende des ersten Dramenteils nach dem Doppelquellwunder befreien kann, macht sie nicht nur selbst in ihrer Rede und ihrem Verhalten deutlich (1127-1130), sondern dies wird zusätzlich noch von Johannes, teilweise sogar in demselben Wortlaut, so interpretiert (1231-1238).

23 Zur Betonung der Körperlichkeit der Leiden ihres Sohnes, bevor Maria in Panik gerät, vgl. auch 920 - 927 u. 1319-1337, 1499f., immer in Medea-Versen.

24 Z. B. 867-871 mit den intertextuellen Einschreibungen in Med.1008, 1042f., 1168. Vgl. hierzu auch 444-450 mit Med. 1008, 1042.

25 Z. B. 884 f. mit den intertextuellen Einschreibungen in Med. 281, 1208. Vgl. hierzu auch 1330-1333 mit Med. 1210, 1183.

26 Z. B. 886-890 mit den intertextuellen Einschreibungen in Med. 1209f., 386-388.

27 Vgl. für die Auffassung von Marias Verzweiflung vor dem Kreuz als maßlos das Urteil Johannes' in 932, 962, 970 - 982. Für weitere Stellen siehe Anm. 22.

28 Es handelt sich hierbei um die auf Joh 19,31-35 zurückgehende, aber vor allem aus Longin bekannte Episode (zum Verhältnis des Christus patiens zu Longin siehe Dölger 1934), dass sich aus der Speerwunde, die ein Soldat dem Toten am Kreuz zufügt, ein unvermischter Doppelstrom aus Blut und Wasser ergießt, durch den dieser den Toten als wahren Sohn Gottes erkennt und sich damit salbt. 
Beim Doppelquellwunder handelt es sich damit um einen signifikanten Einschnitt zwischen dem ersten ,Verzweiflungs-Medea-Teil` und dem zweiten ,Gotteserkenntnis-Bakchen-Teil` des Christus patiens, der auch auf intertextueller Ebene deutlich wird. Denn auf intertextueller Ebene findet das Ende von Marias Verzweiflung darin Ausdruck, dass der erste Christus patiens-Teil in seinen letzten vier Versen kurz nach dem Doppelquellwunder mit einer intertextuellen Einschreibung in die letzten vier Verse der Medea endet, in denen ausgerechnet die Hoffnung auf etwas, das von Gott wider Erwarten gewährt wird, das zentrale Motiv ist (1130 - 1133: Med.1415-1418).

Marias Verzweiflung beim Anblick des leidenden Sohnes, die ihrem eigentlichen Wissen um den Heilsplan entgegensteht, scheint daher mit dem Ende der Medea am Ende des ersten Christus patiens-Teil gewissermaßen zusammenzufallen und infolge des Doppelquellwunders wider Erwarten vom hoffnungsvollen Blick in die Zukunft abgelöst zu werden, der auch am Ende der Medea steht. Damit hat Maria am Ende des ersten Dramenteils das geschafft, was sie den gesamten ersten Teil über versucht hatte: den Kummer über ihre eigentlich so sichere Hoffnung endlich nicht mehr siegen zu lassen. ${ }^{29}$ In der Tat folgt im zweiten Christus patiens-Teil eine Maria, die in BakchenVersen wie unter göttlicher Inspiration die längste Offenbarungsrede des Dramas hält und in Rhesos-Versen voller Hoffnung um ihren Sohn trauert, nicht aber mehr in Medea-Versen daran verzweifelt.

\section{Die intertextuelle Verwendungs- und Wirkungsweise der Bakchen im Christus patiens}

Die ersten Einschreibungen des Christus patiens in die Bakchen finden sich im ersten Bericht des ersten Boten (152-180), in welchem Maria und ihre Freundinnen am Freitagmorgen über die Ereignisse der letzten Nacht aufgeklärt werden. Diese ersten Einschreibungen des Christus patiens in die Bakchen gehören aber nicht zur eigenen Rede des Boten, sondern zu der von ihm wortwörtlich wiedergegeben letzten Rede Jesu an die Jünger auf dem Ölberg, in welcher Jesus den Jüngern seine Wiederkehr von den Toten prophezeit sowie seinen Vater darum bittet, mit seiner Verherrlichung jetzt zu beginnen (161-169: Ba. 1233-1243). Die bejahende Antwort des Vaters an den Sohn und die Jünger sei, so berichtet der Bote weiter, durch ein Donnergrollen erfolgt. Auch darin lässt sich eine Einschreibung in die Bakchen finden (170 f.: Ba. 1088f.).

Im intertextuellen Dialog diese Verse werden erstmalig im Christus patiens der Kreuzestod Jesu und der Tod des Pentheus in Beziehung zueinander gesetzt. Denn zum einen wird Jesu Bitte an seinen Vater in den Worten der rasenden Agaue erzählt, welche damit prahlt, den Löwen getötet zu haben, für den sie ihren Sohn Pentheus hält; zum anderen schreibt sich die donnernde Antwort Gottes in den Ruf des Dio-

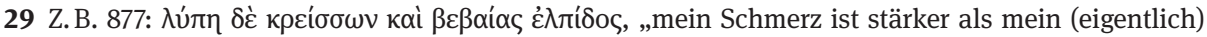
sicheres Hoffen“. Vgl. die ähnlichen Formulierungen in 590 - 597, 720 - 724, 743f., 767-770. 
nysos ein, der die Mänaden zur Tötung des Pentheus antreibt. Unabhängig davon, welche konkreten Folgen sich im Einzelnen für die Interpretation der Textstelle im Christus patiens durch den intertextuellen Dialog mit den Bakchen ergeben, so fällt zunächst auf, dass sich die ersten Einschreibungen des Christus patiens in die Bakchen in den Reden bzw. donnernden Artikulationen zweier göttlicher Instanzen antreffen lassen; dann, dass sich diese Reden göttlicher Instanzen wiederum in Reden von Figuren der Bakchen intertextuell einschreiben, welche zumindest im Moment ihrer Rede göttlich inspiriert sind, nämlich die rasende Agaue und die rasenden Bakchantinnen.

Dass dieser Umstand der göttlichen Inspiration für die Einschreibungen des Christus patiens in die Bakchen bedeutsam ist, bestätigt die nächste (dritte) intertextuelle Einschreibung des Christus patiens in die Bakchen. Diese findet sich in einer an Judas gerichteten prophetischen Rede göttlichen Ursprungs, welche diesem sein herbes Todesschicksal offenbart und ihn zur Umkehr auffordert. In dieser Rede lassen sich an insgesamt drei Stellen intertextuelle Einschreibungen in die Bakchen finden: ganz zu Beginn (191, 193 und 195), in der Mitte (228) und gegen Ende (262-264). In allen drei Christus patiens-Kontexten und den dort aufgerufenen Bakchen-Kontexten geht es darum, dass Judas (im Christus patiens) bzw. Pentheus (in den Bakchen) dazu aufgefordert werden, sich nicht gegen Jesus/Gott/Dionysos zu stellen, weil dieser wahrer Gott und nicht nur ein uneheliches Kind Marias bzw. der Semele sei.

Abermals unabhängig von den konkreten Folgen dieses intertextuellen Dialogs zwischen dem Christus patiens und den Bakchen fällt auch hier auf, dass die Bakchen erneut im Kontext göttlich-prophetischen Sprechens verwendet werden. Es geht auch hier um die Erkenntnis Gottes, welche der jeweilige Sprecher - im Christus patiens der anonyme Sprecher und in den Bakchen der Chor - offensichtlich besitzt und zu welcher er Judas bzw. Pentheus auffordert.

Die vierte intertextuelle Einschreibung des Christus patiens in die Bakchen findet sich in einer Rede, welche Maria nur knapp hundert Verse nach ihrer weiter oben zitierten Medea-Verzweiflungsrede (453-477) hält. ${ }^{30}$ Nach über 500 Versen fast unablässiger Verzweiflung über die Situation zu Beginn des Dramas hält Maria hier ihre nach dem Prolog zweite Rede theologischen Inhalts (568 - 597). Sie erklärt ihren Freundinnen das christliche Mysterium und begründet dabei ihr Verständnis für das Leiden ihres Sohnes als einen Teil des göttlichen Heilsplans mit ihrem Kontakt zum göttlichen Logos, der in ihr „ein Lager aufgeschlagen“ habe (573f.). Wie anders als in jener Verzweiflungsrede klingt Maria hier sowohl in Hinblick auf den Inhalt ihrer Rede als auch in Hinblick auf die intertextuellen Einschreibungen in die Bakchen, wenn sie sagt:

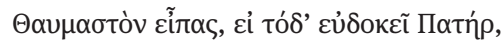

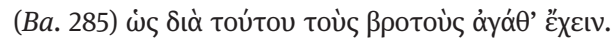

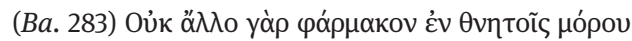

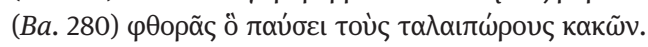

30 Siehe oben S. $400 \mathrm{f}$. 


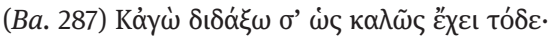

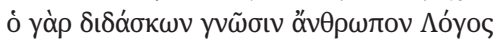

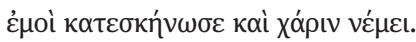

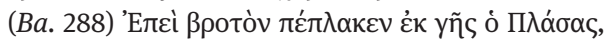

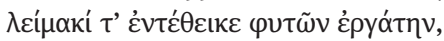

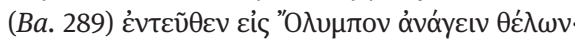

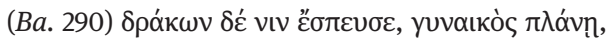

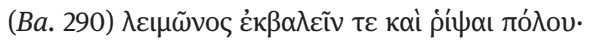

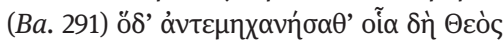

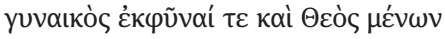

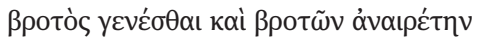

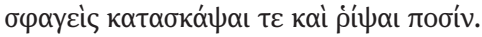

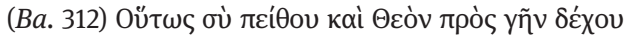

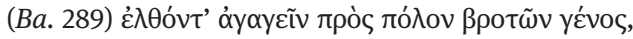

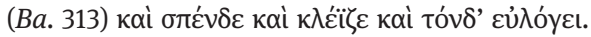

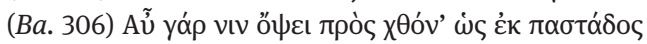

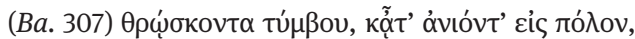

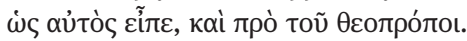

Ein Wunder, meinst du, ist es wenn dies dem Vater so gefällt, dass dadurch (= durch das Leiden Christi) die Menschheit (wieder) gut werde.

Denn kein anderes Heilmittel gegen den Tod gibt es für die Sterblichen,

das die Unglücklichen von Tod und Übel befreien wird.

Und ich will euch darüber unterrichten, dass alles gut so ist.

Denn der Logos ist der Lehrer der menschlichen Erkenntnis

und dieser hat in mir ein Lager aufgeschlagen und mir diese Gnade zugeteilt.

Nachdem der Schöpfer aus Erde den Menschen geschaffen hatte,

da setzte er ihn als Gärtner in den Garten,

weil er ihn von dort zum Himmel hinaufführen wollte.

Aber die Schlange plante eifrig, ihn durch die Verführung der Frau

aus dem Paradies zu vertreiben und vom Himmel fernzuhalten.

Dagegen ersann Gott das Heilmittel,

aus einer Frau zu wachsen und, Gott bleibend,

Mensch zu werden und den Menschenmörder,

indem er selbst ermordet wird, zu zerstören und mit Füßen zu zertreten.

Daher hab' Vertrauen und glaub' an Gott, der auf die Erde

kam, um das Geschlecht der Menschen zum Himmel zu führen.

Ihm spende Opfer, ihm singe Lobgesänge, ihn preise.

Denn du wirst ihn wieder auf Erden sehen, wirst sehen, dass

er sich vom Grab wie von einem Ruhebett erhebt und dann zum Himmel auffährt,

wie er selbst gesagt hat und vor ihm die Propheten. (CP 568-589)

Hatte Maria zuvor entweder das Leiden ihres Sohnes kategorisch ausgeschlossen (111119; 439f.) oder war, nachdem sie es zum ersten Mal mit eigenen Augen gesehen hatte, daran in Medea-Versen dermaßen verzweifelt, dass sie an der Wahrhaftigkeit der Prophezeiungen insgesamt zu zweifeln begann (z. B. 444-450), so scheint Maria hier wie verwandelt, wenn sie ihren Freundinnen das Leiden ihres Sohnes als Teil des göttlichen Heilsplans darzulegen vermag. Maria befindet sich offenbar in einem $\mathrm{Zu}$ stand, in welchem ihr Verstand oder Geist Einblick in den göttlichen Logos, den Lehrer 
aller in allem, hat. Wie unter göttlicher Inspiration gelingt es Maria hier, nicht nur selbst das Paradoxon des leidenden Gottes geistig zu durchdringen, sondern auch, es

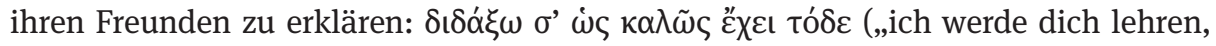
dass sich dies gut verhält“, 272). Es handelt sich hierbei um ein Zitat aus der TeiresiasRede (Ba. 287), mit welchem dort Teiresias den Pentheus über die Wahrhaftigkeit von Dionysos' Gott-Sein unterrichtet.

Nicht zuletzt also auch auf der Ebene der intertextuellen Einschreibungen kann so deutlich werden, dass Maria hier prophetisch-offenbarenden Zugang zum Göttlichen hat, der sie das momentan sichtbare Leiden ihres Sohnes nicht mehr nur als das sehen lässt, was es im Moment (auch, aber nicht nur) ist, sondern auch als das Leiden des Sohnes Gottes zum Heil der Menschheit. Denn der Teiresias aus den Bakchen, in dessen Worte der Dichter sich diese Maria einschreiben lässt, verfügt als Seher grundsätzlich über den Zugang zur göttlichen Sphäre, hat darüber hinaus in dieser konkreten Situation Dionysos als Gott erkannt, wie Fellkleidung, Tänze und Thyrsos offenbaren und ruft daher Pentheus dazu auf, sein Unverständnis zu beenden und Dionysos als Gott anzuerkennen (Ba. 265-329) - wie auch die Maria im Christus patiens ihre Freundinnen, den Chor, ermahnt.

Damit wird die Erwartungshaltung bezüglich der intertextuellen Wirkung bestätigt, welche bereits durch die ersten beiden Einschreibungen des Christus patiens in die Bakchen aufgebaut worden ist. Denn alle bisherigen Einschreibungen in die Bakchen ließen sich ausschließlich in Reden göttlichen Ursprungs finden: sei es, dass Jesus um den Beginn seiner Verherrlichung bittet und den Jüngern seine Auferstehung prophezeit, sei es, dass Gott seinem Sohn und den Jüngern ein bestätigendes Zeichen sendet oder dass Judas von einer göttlichen Stimme zur Umkehr und zur Gotteserkenntnis ermahnt wird oder dass Maria ihren Freundinnen das christliche Mysterium offenbart.

Auch die fünfte Einschreibung in die Bakchen, welche als letzte noch vor dem zweiten sogenannten Bakchen-Teil liegt, reiht sich bezüglich ihrer Wirkung, eine Rede unter göttlicher Inspiration zu sein, hierin ein. Diese befindet sich in der Rede des dritten Boten, der sich als Johannes zu erkennen gibt. Johannes' Bericht schreibt sich dominant in die Bakchen ein, und zwar hier in jenen Botenbericht, welcher von der Ermordung des Pentheus berichtet (bes. Ba. 1043-1099). Dadurch wird der in der ersten Bakchen-Einschreibung aufgemachte Dialog zwischen Jesu Kreuzestod und der Ermordung des Pentheus durch die Mänaden (161-172) hier wieder aufgenommen, als Johannes von Jesu Gang nach Golgatha, von seiner Kreuzigung, vom Schlagen des Gekreuzigten, vom Essigschwamm und von der Reaktion der Umstehenden berichtet (657-681).

Als tertium comparationis zwischen dem Tod Jesu und dem des Pentheus scheint hier nun deutlicher als zuvor auf, dass sowohl Pentheus als auch Jesus nach dem Plan Gottes in der Absicht getötet bzw. geopfert werden, um das wahre Gott-Sein (des Dionysos bzw. Jesu als wahrer Sohn Gottes) und die Herrlichkeit seiner Macht auf Erden zu offenbaren. Als konkrete Folge dieses intertextuellen Dialogs zwischen Johannes' Rede im Christus patiens mit der Rede des Boten in den Bakchen ergibt sich, 
dass Johannes’ Bericht von der Kreuzigung Jesu im Christus patiens eine Offenbarungsfunktion zukommt, insofern als der Bericht über die Tötung des Pentheus in den Bakchen genau dies ist: die am Ende des Dramas stehende Offenbarung von Dionysos' wahrem Gott-Sein mittels der Tötung eines Menschen, das anzuerkennen und hinfort zu respektieren allen geboten wird. ${ }^{31}$

Worum es also bei den intertextuellen Einschreibungen des Christus patiens in die Bakchen daher allgemein zu gehen scheint, sind zum einen die Art und Weise des Sprechens - im weitesten Sinne: unter göttlicher Inspiration -, zum anderen der Inhalt der Rede - im weitesten Sinne: Gotteserkenntnis und -offenbarung. Vor diesem Hintergrund kann auch erklärt werden, warum die Bakchen im zweiten Teil des Christus patiens, in welchem im Gegensatz $\mathrm{zu}$ allen anderen Teilen die Gotteserkenntnis und die prophetische Gottesoffenbarung der Maria (1489-1619) (und des Johannes: 1637-1699) die vorrangigen Themen sind, so prominent rezipiert werden: weil hier nach der in Medea-Versen verzweifelnden Maria des ersten Dramenteils endlich eine Maria zu sehen ist, welche in ihrer längsten Rede des Dramas (14891619) vor allem in den Worten des Dionysos aus dem Bakchen-Prolog die Göttlichkeit ihres toten und daher in diesem Moment eigentlich am wenigsten göttlich erscheinenden Sohnes allen offenbart bzw. infolge des Doppelquellwunders allen (wieder) offenbaren kann, die sich wie sie selbst noch im ersten Dramenteil von diesem sinnlichen Wahrnehmungseindruck zu sehr leiten und dadurch in die Irre führen lassen.

\section{Die intertextuelle Verwendungs- und Wirkungsweise des Rhesos im Christus patiens}

Bei den ersten Einschreibungen des Christus patiens in den Rhesos zu Beginn des Dramas fällt auf, dass für die Erwähnung der im Christus patiens herrschenden Dunkelheit wiederholt auf die im Rhesos erwähnte Dunkelheit zurückgegriffen wird. ${ }^{32}$ Beide Dramen beginnen bei Nacht: einer Nacht im letzten Jahr des Trojanischen Krieges im Feldlager der Troer bzw. der Nacht, welche zwischen dem Donnerstag, da

31 In der Retrospektive kann eine ähnlich gerichtete Wirkung auch für die ersten beiden Einschreibungen des Christus patiens in die Bakchen (161-172) konstatiert werden. Das waren zum einen Jesu Bitte an den Vater, sogleich mit seiner Verherrlichung zu beginnen, und zwar in den Worten der Agaue, welche sich rühmt, einen Löwen erlegt zu haben, zum anderen der bestätigende Donner Gottes in den Worten, mit denen Dionysos die Mänaden zur Ermordung des Pentheus antreibt. Beide intertextuellen Einschreibungen geben damit auf der Ebene des intertextuellen Dialogs den bevorstehenden Tod Jesu als Offenbarung der Macht und des wahren Gott-Seins Gottes zu erkennen, da ja auch der Tod des Pentheus am Ende der Bakchen die Macht und das wahre Gott-Sein des Dionysos offenbart.

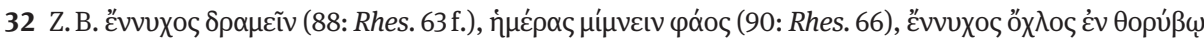

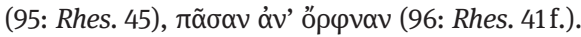


Jesus im Garten Getsemani verhaftet wird, und dem Freitag liegt, an dem Jesus auf Golgatha vor den Toren der Stadt Jerusalems gekreuzigt wird.

Vergleichbar ist auch die Situation zu Beginn beider Dramen, wobei sowohl die Figurenpaare - Maria und Chor im Christus patiens bzw. Hektor und Chor im Rhesos als auch ihre Reden miteinander vergleichbar gestaltet sind: Wie Hektor und seine Wache (= der Chor) zu Beginn des Rhesos, so sehen auch Maria und ihre Freundinnen (= der Chor) zu Beginn des Christus patiens in der Ferne - in Jerusalem bzw. im Schiffslager der Griechen - eine Menschenmenge zusammenkommen, deren Fackeln in der Dunkelheit weithin sichtbar sind, und fragen sich, was das zu bedeuten habe.

Ähnlich wie Hektor, der noch bei Nacht ins Schiffslager der Griechen einzufallen begehrt, weil er die nächtliche Versammlung der Griechen als Fluchtvorbereitungen (miss)deutet, so wünscht sich auch Maria, noch bei Nacht nach Jerusalem zu laufen, um mit eigenen Augen zu sehen, welches Leid ihr Sohn dort erdulden müsse; und ähnlich wie Hektor von seiner Wache, so wird auch Maria von ihren Freundinnen mit einem Hinweis auf die unkalkulierbare Gefahrenlage dazu überredet, den nächtlichen Gang nach Jerusalem bzw. im Falle Hektors den nächtlicher Angriff auf das griechische Schiffslager auf den morgigen Tag zu verschieben.

Eine mögliche Wirkung dieses intertextuellen Dialogs ergibt sich nun daraus, dass neben dieser offensichtlichen Situationsähnlichkeit - die/der Protagonist(in) begehrt zu Beginn des Dramas eine nächtliche Aktivität, wird aber vom Chor der jeweiligen Tragödie unter Hinweis auf die Gefahrenlage davon abgebracht - auch das weniger offensichtliche Motiv der Ungeduld, mit welcher im Rhesos von Beginn an das Licht des Tages erwartet wird, erstmals intertextuell aufgerufen wird und dadurch auch für den Christus patiens aktiviert werden kann. ${ }^{33}$ Für den Leser eines so verstandenen intertextuellen Dialogs würde somit die Marienfigur des Christus patiens zu Beginn des Dramas den Anbruch des Tages nicht nur deswegen herbeisehnen, um dann endlich gefahrlos(er) erfahren zu können, wie es ihrem Sohn in Jerusalem ergeht, sondern auch deswegen, weil sie wie Hektor zu Beginn des Rhesos erwartet, dass dann der Tag der Rettung der Menschheit wie der Trojas aus Leid und Tod endlich anbricht.

In der Tat lässt sich dieses Motiv, das Licht des (dritten) Tages als Rettung nach dem nächtlichen Dunkel zu erwarten, als ein zentrales Wirkungsmotiv des intertextuellen Dialogs zwischen Christus patiens und Rhesos herausarbeiten. Die Sehnsucht nach dem kommenden Tag als dem Tag der Rettung aus Krieg, Leid und Tod ist dabei nicht nur im Rhesos allgegenwärtig, ${ }^{34}$ sondern gehört auch im Christus patiens zur Grundstimmung des Dramas, in dessen Verlauf alle Figuren immer wieder das Licht der (dritten) Sonne herbeisehnen, weil sich mit deren Erscheinen die Rettung der Menschheit gemäß dem göttlichen Heilsplan endlich erfüllt haben werde. ${ }^{35}$ In beiden

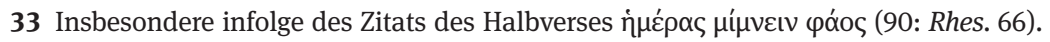

34 Z.B. Rhes. 367-370, 464-466. Vgl. auch 535f., 600-605, 614f., $984 \mathrm{f}$.

35 Im Christus patiens liegt die Hoffnung aller auf dem Kommen des glücklichen, dritten Tages

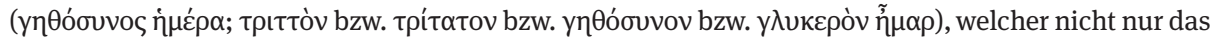


Tragödien kommt dabei der sich immer wiederholenden sprachlichen Thematisierung von Hoffnung, Erwartung, Sehnsucht, Ungeduld, welche mit dem Anbruch des (dritten) Tages verbunden sind, eine spannungssteigernde Funktion zu, etwa in dem Sinne, dass sich dadurch beim Leser die Frage einstellen kann, ob sich diese Hoffnung im Drama wohl erfüllen möge oder unerfüllt bliebe und wie es dazu kommen werde.

Wie der Rhesos, so spielt dabei auch der größte Teil des Christus patiens bei Dunkelheit. ${ }^{36}$ Zahlreich sind daher im Christus patiens die Ausdrücke, mit denen immer wieder auf die im Drama herrschende Dunkelheit verwiesen wird. ${ }^{37}$ In diesem Zusammenhang fällt auf, dass alle drei Christus patiens-Teile bei Dunkelheit beginnen - und zwar jeweils mit dem Zitat von Rhesos-Versen, welche dort die Dunkelheit thematisieren. So setzt wie der erste Teil auch der zweite Teil des Christus patiens, obwohl er eigentlich bei Tage spielt, weil Jesus am Freitag bei Tage gekreuzigt wird, deshalb im Dunkeln ein, weil sich mit dem Tode Jesu die Sonne verdunkelt; außerdem macht er in seinem ersten Vers die momentan herrschende Dunkelheit über eine intertextuelle Einschreibung in den Beginn des Rhesos deutlich (1134 : Rhes. 85f.). Und auch der dritte Teil des Christus patiens beginnt am frühen Sonntagmorgen noch bei Dunkelheit und thematisiert diese unter Verwendung intertextueller Einschreibungen in den Rhesos auch sprachlich. ${ }^{38}$ Man darf den Christus patiens daher als eine Tragödie bezeichnen, die wie der Rhesos prinzipiell bei Dunkelheit spielt.

Allerdings - die einzige Szene im Christus patiens, welche in explizitem Gegensatz zum Rhesos, der kurz vor oder gerade mit Tagesanbruch endet, bei hellem und gleißendem Sonnenlicht spielt, ${ }^{39}$ und in welcher immer wieder das helle und gleißende

Licht, sondern der Menschheit die Rettung bringe, z.B.: 780f., 891-894, 954f., 957-961, 979-982, 1191f., $1480 \mathrm{f} ., 1785 \mathrm{f}$.

36 Die Handlung des Christus patiens beginnt in der Nacht von Donnerstag zu Freitag (92f.); spielt am Freitag, an welchem sich beim Tode Jesu die Sonne verdunkelt (872f., vgl. auch 993f., 1205); bringt die Kreuzabnahme und die Beerdigung Jesu auf die Bühne, welche bei einbrechender bzw. voller Dunkelheit stattfinden - vor der Kreuzabnahme drängt Maria zur Eile, weil die Nacht hereinbreche (1474f.), nach der Beerdigung ist, laut Johannes, die Nacht schon weit vorgerückt (1815-1817) -; lässt den Sonntag noch vor Dämmerung bei Dunkelheit beginnen sowie am Abend bei Dunkelheit enden ( $2173 \mathrm{u}$. 2176f.), in welcher ein Bote Maria von dem Verrat der Hohepriester berichtet (2194-2388), Maria Magdalena von ihren Erlebnissen mit Johannes und Simons Petrus am Grab erzählt (2437-2479) und die Frauen zu den elf im Hause der Mutter des Markus versammelten Jüngern dazustoßen (2480 - 2531), wo der Auferstandene erscheint und die letzte Rede des Dramas hält.

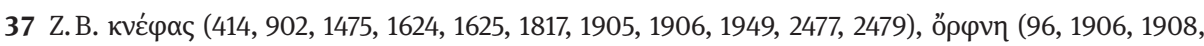

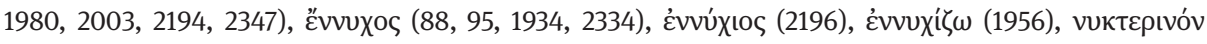

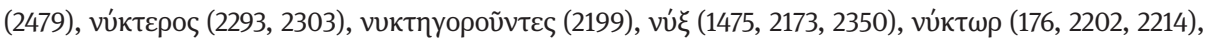

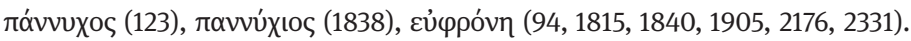

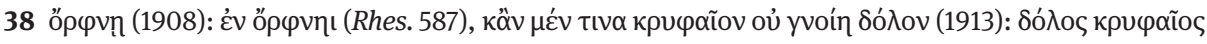

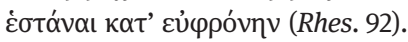

39 Zwar beginnt auch der Samstag bei Tage (1843-1846), doch wird er innerhalb des dramatischen Geschehens im Christus patiens nicht weiter thematisiert. Maria und ihre Freundinnen verbringen den Samstag im Hause des Johannes, ohne dass etwas davon auf der Bühne dargestellt wird. Die Handlung des Christus patiens setzt erst Sonntagnacht kurz vor Morgendämmerung wieder ein (1906). 
Licht des Tages auch sprachlich thematisiert wird, ist die zu Beginn des dritten Dramenteils liegende sonntägliche Szene am leeren Grab, in welcher der Wiederauferstandene, der immer wieder im Drama als der Lichtbringer bezeichnet wird, ${ }^{40}$ seiner Mutter Maria und der Maria Magdalena erscheint (2020 - 2170). In dieser Szene wird nicht nur das Licht der Sonne vor Jesu Erscheinen von Maria und Maria Magdalena abermals sehnsüchtig erwartet, sondern auch die ersten zaghaft am Horizont erscheinenden Sonnenstrahlen sowie das hell und gleißend am Himmel stehende Sonnenlicht werden in begeisterten Jubelreden stürmisch begrüßt, weil es endlich die Wiederauferstehung des Toten und die Rettung der Menschheit gebracht habe. ${ }^{41}$

Bemerkenswert ist hierbei nun, dass der Rhesos im Christus patiens offensichtlich nicht nur in Hinblick auf die im Christus patiens wie im Rhesos herrschende Dunkelheit und die in beiden Tragödien gleichermaßen herrschende Sehnsucht auf den folgenden Tag als den Tag der Rettung rezipiert wird, sondern, dass sich der Christus patiens auch ausgerechnet zu Beginn seines dritten Teils so dominant intertextuell in den Rhesos einschreibt, in welchem sich die Hoffnung Marias und ihrer Freundinnen erfüllt und der Tag der Rettung endlich anbricht. Bemerkenswert ist dies deshalb, weil der Tag der Rettung im Rhesos selbst erscheint: Das Drama endet noch vor Sonnenaufgang, und mit Rhesos stirbt jegliche Hoffnung auf die Rettung Trojas. ${ }^{42}$ Hektor und seine Wache aber geben nicht einmal am Ende des Rhesos die Hoffnung darauf auf (991f.).

Damit macht der dritte Teil des Christus patiens, der sich so dominant in den Rhesos einschreibt, schließlich aus dem Rhesos, in dessen dramatischen Verlauf die Dunkelheit selbst nicht überwunden wird, die Hoffnung auf Rettung am nächsten Tag sich als eitel, ja die Rettung grundsätzlich als nie mehr erreichbar erweist, gleichsam ein Drama des gleißenden Lichtes, der sicheren Hoffnung und der sich schließlich auch wirklich erfüllenden Rettung. In diesem Sinne kann der Rhesos als eine Tragödie, in der sich die Hoffnung auf das Erscheinen der Sonne und die damit verbundene Rettung nicht erfüllt, ihre Wirkung im Christus patiens entfalten, und zwar in dem Sinne, dass der dritte Christus patiens-Teil den Rhesos mit positiver Umkehrung fortschreibt: Die Sonne, die dort fehlt, geht hier endlich auf, und der Tag der Rettung, der dort nie mehr kommen kann, bricht hier schließlich an.

Vor dem Hintergrund dieser intertextuellen Lesart verwundert es nicht, dass der Jesus des Christus patiens zum Rhesos wird - die Lautähnlichkeit der Namen Jesus/ Rhesos ist ein schöner Nebeneffekt -, der wie Rhesos zwar spät, d.h. erst nach einer langen Serie von Bitten zum Kampf mit dem Feind seit Abraham $(202,1368)$ erschienen ist, und dem wie Rhesos ein einziger Tag reicht, um den Kampf mit dem Feind zum Erfolg zu führen und die Rettung aller von Leid und Tod zu bringen, der aber dann anders als Rhesos - diese Hoffnung auch erfüllen können wird, weil er die Nacht und

40 Vgl. 1513f., 1537f., 2027-2029.

41 Z.B. 2020 f., 2024f., 2029f., 2076f, 2108-2110.

42 Athena hatte ja deutlich gemacht (Rhes. 605), er sei der einzige Retter Trojas. 
den Tod übersteht und als Licht der Rettung am nächsten Tag der Welt erscheint, so etwa in einer Rede des Johannes:

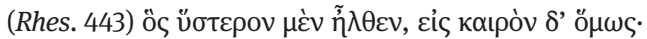

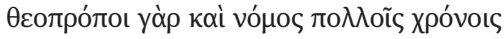

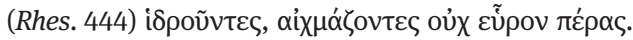

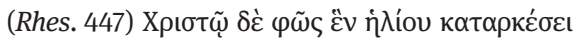

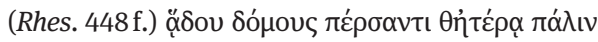

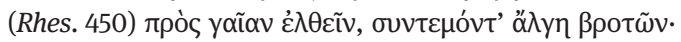

Er ist spät gekommen, doch zur rechten Zeit.

Denn die Propheten und das Gesetz mühten sich viele Jahre,

in dem Kampf fanden sie aber kein Ende.

Christus aber genügt ein einziges Licht der Sonne,

um die Häuser des Hades zu zerstören und am selben Tag wieder

auf die Erde zurückzukommen und das Leiden der Menschheit zu beenden. (1728-1730)

Eine grundsätzliche Wirkung des intertextuellen Dialogs zwischen Christus patiens und Rhesos scheint sich folglich von einem Leser dahingehend konkretisieren zu lassen, dass aus dem Rhesos, dem paganen Drama der Nicht-Erfüllung der Hoffnung auf Rettung aus Leid und Tod, im Christus patiens das christliche Drama der Erfüllung der Hoffnung auf Rettung aus Leid und Tod wird. Den Tag der Freiheit, welchen Hektor am Ende des Rhesos zwar erneut, aber, wie der Rezipient weiß, vergeblich, in Aussicht stellt (Rhes. 991f.), genau diesen bringt der Christus patiens, den Rhesos gleichsam fortund umschreibend, endlich auf die Bühne.

Für diese Deutung der grundsätzlichen Wirkung des intertextuellen Dialogs zwischen Christus patiens und Rhesos ist bezeichnend, dass die letzten Verse des Hektor im Rhesos, in denen er abermals vergeblich die Hoffnung formuliert, der einbrechende Tag werde den Troern endlich die Rettung bringen (Rhes. 990 f.), gleich zweimal im Christus patiens zitiert werden: Das eine Mal spricht Johannes sie im zweiten Dramenteil (1783f.), das andere Mal Maria im dritten Dramenteil und hier relativ zu Beginn, kurz bevor die Sonne im Christus patiens im Gegensatz zum Rhesos aufgeht und der Tag der Rettung hier wirklich erscheint:

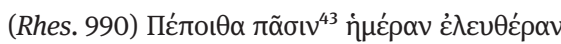

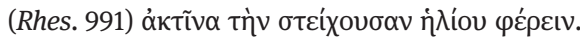

Ich bin davon überzeugt, dass allen den Tag der Freiheit

die aufsteigenden Strahlen der Sonne bringen. (2010f. = 1783f.)

Wenn im Christus patiens die Figur des Johannes, der durchweg im Drama nicht nur der „Theologe“ heißt, sondern auch in all seinen Reden und Handlungen einen weitreichenden Einblick ins göttliche Heilsgeschehen, ein tiefgreifendes Verständnis der göttlichen Zusammenhänge sowie allgemein große Gottesfürchtigkeit zur Schau

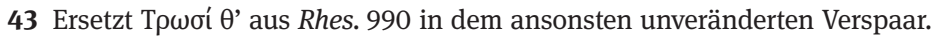


stellt, im zweiten Dramenteil noch vor dem verschlossenen Grab diese letzten und im Rhesos eitlen Worte Hektors aufgreift, so wirkt dies in zwei unterschiedliche Richtungen. Auf der einen Seite lässt hierbei nämlich Johannes’ große Autorität im Christus patiens vermuten, dass er anders als Hektor auf etwas hofft, was sich dann auch wirklich erfüllen wird; gleichzeitig wirkt auf der anderen Seite das Rhesos-Zitat an dieser Stelle im Christus patiens auch spannungssteigernd, insofern wenigstens implizit die Frage aufkommt, ob denn im Christus patiens die Hoffnung wie im Rhesos unerfüllt bleiben oder sich im Gegensatz zu diesem doch erfüllen werde.

Wenn dann das zweite Mal die Figur der Maria im Christus patiens dieselben Worte zu Beginn des dritten Dramenteils spricht und kurz darauf die Sonne wirklich aufgeht, dann hat sich hier endlich das erfüllt, was zwar wie im Rhesos das ganze Drama hindurch erwartet wird, dort jedoch unerfüllt bleibt. Vor diesem Hintergrund handelt es sich also beim dritten Teil des Christus patiens um nichts anderes als um die kontrastive Fortschreibung des Rhesos, insofern der Beobachtung der Morgendämmerung im Christus patiens wie im Rhesos (1950, 1963 u. 1997f.: Rhes. 985) die Formulierung der Hoffnung in beiden Tragödien (2010: Rhes. 991f.) folgt, dann aber im Rhesos die Tragödie endet, wohingegen im Christus patiens nur wenige Verse später das Licht der Sonne wirklich erscheint (2019-2021) und mit ihm der Retter der Menschheit.

Der Rhesos wird daher vom Christus patiens als Tragödie der Hoffnung rezipiert, die sich allerdings erst im christlichen Drama mit seinem christlichen Retter erfüllen kann. Eine Maria, die im zweiten Dramenteil mit dem Leichnam ihres Sohnes im Arm in Rhesos-Versen trauert (1337-1430), macht daher durch die Verwendung des Rhesos als Referenztext deutlich, dass sie von einer Maria, die noch im ersten Dramenteil in Medea-Versen verzweifelt war, weit entfernt ist. Im intertextuellen Dialog offenbart nämlich diese in Rhesos-Versen trauernde Maria des zweiten Dramenteils, dass ihre Hoffnung trotz momentaner Trauer, die sie als seine Mutter an den Tag legt, nach dem Doppelquellwunder wieder eine grundsätzlich sichere geworden ist, die sich nicht mehr wie noch im ersten Dramenteil von einer überbordenden Verzweiflung besiegen lässt.

Vor dem Hintergrund dieser Deutungen der intertextuellen Dialoge des Christus patiens mit dem Rhesos und deren Wirkung für den Christus patiens insgesamt lassen sich also tatsächlich bereits die ersten Einschreibungen des Christus patiens in den Rhesos so verstehen, dass Maria zu Beginn des Christus patiens wie Hektor zu Beginn des Rhesos den folgenden Tag primär deswegen herbeisehnt, weil sie wie er darauf hofft bzw. erwartet, dass dann der Tag der Rettung der Menschheit bzw. Trojas aus Leid und Tod anbricht. 


\section{Zusammenfassung: Warum steht eine ,Maria-Medea' im Zentrum des Christus patiens?}

Die vorangehende Darstellung der intertextuellen Verwendungs- und Wirkungsweisen der intertextuellen Dialoge des Christus patiens mit der Medea, den Bakchen und dem Rhesos kann folgendermaßen zusammengefasst werden:

1) Die intertextuellen Einschreibungen des Christus patiens in den Rhesos verweisen immer wieder sowohl auf die momentan herrschende Dunkelheit als auch die erhoffte Rettung am folgenden Tag und deuten dadurch spannungssteigernd auf den dritten und letzten Teil des Dramas voraus, wo schließlich nicht nur die meisten intertextuellen Einschreibungen in den Rhesos zu finden sind, sondern wo auch, anders als im Rhesos, die über weite Teile herrschende Dunkelheit vom Licht und vom Heil der dritten Sonne abgelöst wird.

2) Den intertextuellen Einschreibungen in die Medea, welche den ersten Teil des Christus patiens mit Marias Verzweiflung dominieren, kommt die Wirkung zu, Marias verzweifeltes Sprechen als quasi-erotischen Wahnsinn zu der in Maria durch die konkreten Wahrnehmungseindrücke ihres leidenden Sohnes jeweils ausgelöst wird. Denn unter diesen Wahrnehmungseindrücken ist Maria jeweils unfähig das momentane Geschehen als etwas anderes zu verstehen, als was es ihr gerade zu sein scheint. Eine Folge davon ist, dass der ihr eigentlich zur Verfügung stehende, verstandesgemäße Einblick in den göttlichen Heilspan und ihre darauf basierende so sichere Hoffnung auf dessen Erfüllung vom Bedürfnis, ohne Verstand zu weinen, überwältigt werden.

3) Im Gegensatz dazu weisen die intertextuellen Einschreibungen in die Bakchen, derer sich Maria (und Johannes) insbesondere im zweiten Teil des Christus patiens in den großen Offenbarungsreden vor dem verschlossenen Grab bedienen, auf ihre göttliche Inspiration und ihren verstandesgemäßen Zugang zum göttlichen Logos hin, aus dem schöpfend sie auch anderen das christliche Mysterium darlegen können.

Was also die Rezeption der drei Euripides-Tragödien anbelangt, so lässt sich insgesamt festhalten, dass der Christus patiens den Rhesos als Tragödie der unvollendeten Hoffnung auf Heil und Rettung, welche erst im Christus patiens erfüllt wird, rezipiert, die Medea als Tragödie des in ,erotischem' Wahnsinn und ohne Verstand handelnden Menschen, der ganz unter dem Eindruck des sinnlich Wahrnembaren steht, und die Bakchen als Tragödie der Gottesoffenbarung und Gotteserkenntnis eines Menschen, dessen Verstand/Geist ganz auf Gott gerichtet ist.

Dass im ersten Teil des Christus patiens, dem mit insgesamt 1133 Versen längsten Dramenteil, eine ,Maria-Medea' so dominant ist, scheint mit der Wirkungsabsicht des Christus patiens als christlichem Drama insgesamt zusammenzuhängen. Die Wirkungsabsicht des christlichen Dramas lässt sich wiederum damit beschreiben, dass Marias spezifische Darstellung im Christus patiens auf die Rolle vorbereiten solle, in welcher sie im letzten Gebet des Dramas (2572-2602) vom Gebetssprecher angerufen 
wird, nämlich: in die Rolle der Fürbitterin des Gläubigen bei ihrem Sohn im Himmel sowie der Retterin des Sünders aus allen Nöten.

Gerade die intertextuellen Dialoge können hierbei deutlich machen, inwiefern Maria diese ihr im Mariengebet zugedachte Rollen besonders gut erfüllen kann. Denn zum einen teilt sich Maria infolge der intertextuellen Einschreibungen mit der Eva aus dem Dramenprolog ein sogenanntes ,Medea-Element‘. Dieses besteht darin, sich vom sinnlichen Wahrnehmungseindruck (ihres Sohnes wie des Apfels) in eine solche Raserei versetzen zu lassen, dass eine verstandesmäßig angemessene Reaktion (nicht zu verzweifeln bzw. nicht nach dem Apfel zu greifen) nicht mehr oder nur noch sehr schwer möglich ist. Als eine solche Maria wird sie wiederum ganz zum Kind Evas (und Adams) und ist damit zunächst einmal - so wie jeder andere Mensch auch - ganz Mensch. Zum anderen aber verfügt sie im Christus patiens, wie sie immer wieder insbesondere in ihren Bakchen-dominierten Reden unter Beweis stellt, über einen exzeptionellen Einblick in den göttlichen Heilsplan und versteht Dinge, die anderen, in welchen der göttliche Logos „kein Lager aufgeschlagen“ (574) hat, verschlossen bleiben (müssen).

Eine solche Marienfigur, die im Christus patiens quasi sowohl ,Medea“ in ihrer Verzweiflung als auch, Teiresias‘ in ihrer Gotteserkenntnis ist, scheint auf die „wohlwollende“ (عũ $\varphi \rho o v o v ̃ \sigma \alpha, 2601)$ „Retterin des Sünders aus allen Gefahren“

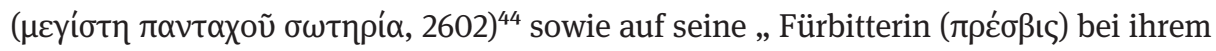
Sohn“ (2589), als welche sie zuletzt im Christus patiens angerufen wird, besonders gut vorzubereiten. Denn wer könnte besser den Sünder aus allen Gefahren retten und bei ihrem Sohn für ihn Fürbitte einlegen als eine Maria, die wie Eva und wie grundsätzlich jeder andere Mensch den durch einen sinnlichen Eindruck ausgelösten ,erotischen“ Wahnsinn selbst erlebt, ihn aber durch die geistige Rückbesinnung auf das Göttliche erfolgreich überwunden hat?

Von einer solchen Maria, wie sie der Christus patiens konstruiert, nicht zuletzt auch durch seine intertextuellen Dialoge mit der Medea für eine Maria, die am sinnlichen Wahrnehmungseindruck vom leidenden Sohn gänzlich zu verzweifeln droht, mit den Bakchen für eine Maria, die sich auf die göttliche Wahrheit rückbesinnt, und mit dem Rhesos für eine Maria, deren Glaube an die Erfüllung des göttlichen Heilsplanes dadurch (wieder) unerschütterlich geworden ist - von einer solchen Maria darf man erwarten, dass sie dem Sünder, der sie anruft, aufgrund ihrer eigenen Erfahrung sowohl mit Wohlwollen begegnet als auch als Vorbild für seine eigene Rückbesinnung auf Gott dient. Es scheint hierin die allgemeine Wirkungsabsicht des Christus patiens $\mathrm{zu}$ liegen und der Grund dafür, warum Maria die eigentliche Zentralgestalt dieses christlichen Dramas und seine Identifikations- und Leitfigur für den Rezipienten ist. Dies ist nicht nur Ausdruck einer bestimmten Werkintention, sondern auch einer bestimmten Mariologie und Ekklesiologie. Diese zu rekonstruieren, muss jedoch Gegenstand einer anderen als einer literaturwissenschaftlichen Untersuchung sein.

44 Vgl. 2858, 2588, 2598. 


\section{Literatur:}

Augusti 1855: Johann Christian W. Augusti, „Mit welchem Rechte wird das theologische Drama,

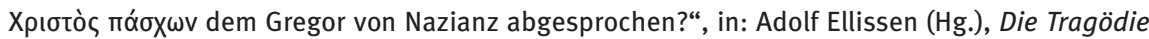

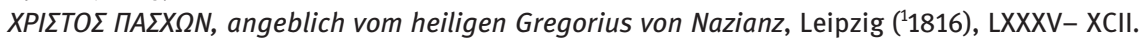

Binder 1981: Wilhelm Binder, Euripides. Sämtliche Tragödien und Fragmente in 6 Bänden. Band 6: Fragmente, der Kyklop, Rhesos. Fragmente übs. von Gustav Adolf Seeck, Der Kyklop übs. von Johann Jakob Christian Donner, Rhesos übs. von W. B., Darmstadt.

Brambs 1884: Johann Georg Brambs, De auctoritate tragoediae Christianae, quae inscribi solet

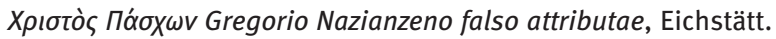

Dölger 1934: Franz Dölger, „Die Blutsalbung des Soldaten mit der Lanze im Passionsspiel Christus Patiens. Zugleich ein Beitrag zur Longinus-Legende“, Antike und Christentum 4, 81-94.

Ebener 2010: Dietrich Ebener, Euripides. Ausgewählte Tragödien. In zwei Bänden. Griechisch und deutsch. Aus dem Griechischen von D. E. Hrsg. von Bernhard Zimmermann, Mannheim.

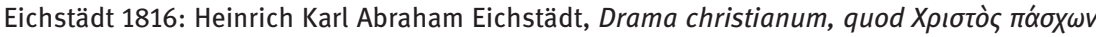
inscribitur, num Gregorio Nazianzeno tribuendum sit, Eichstätt.

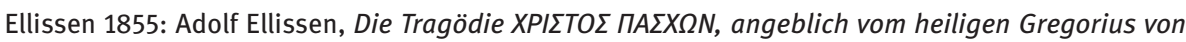
Nazianz. Im Originaltext und zum ersten Mal in metrischer Verdeutschung mit literar-historischer Einleitung und erläuternder Analyse, Leipzig.

Glei u. Natzel-Glei 1996: Reinhold Glei und Stephanie Natzel-Glei 1996, Apollonios von Rhodos: Das Argonautenepos. Hrsg., übs. und erl. von R. G. u. S. N.-G., Darmstadt.

Hunger 1978: Herbert Hunger, Die hochsprachliche profane Literatur der Byzantiner, 2 Bände, München.

Most 2008: Glenn W. Most, „On the Authorship of the 'Christus Patiens'“, in: Andrea Jördens u.a. (Hgg.), Quaerite faciem eius semper. Studien zu den geistesgeschichtlichen Beziehungen zwischen Antike und Christentum, Hamburg, 229-40.

von Schirnding 2007: Albert von Schirnding, Hesiod. Theogonie, Werke und Tage.

Griechisch-deutsch, hrsg. und übs. von A. von S., mit einer Einführung und einem Register von Ernst G. Schmidt, Düsseldorf.

Swart 1990: Gerhard Jacob Swart, A historical-critical evaluation of the play Christus patiens, traditionally attributed to Gregory of Nazianzus, Diss. University of Pretoria.

Thornton 1997: Bruce S. Thornton, Eros: The Myth of Ancient Greek Sexuality, Boulder.

Trisoglio 1974: Francesco Trisoglio, „Il Christus patiens. Rassegna delle attribuzioni“, Rivista di Studi Classici 22, 351- 423.

Tuilier 1969: André Tuilier, Grégoire de Nazianze. La Passion du Christ. Introduction, texte critique, traduction, notes et index par A. T., Paris.

Vakonakis 2011: Nikolaos Vakonakis, Das griechische Drama auf dem Weg nach Byzanz. Der euripideische Cento „Christos Paschon“, Tübingen.

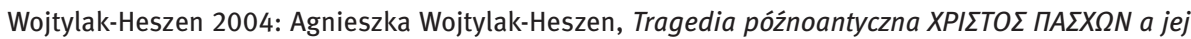
źródta klasyczne, Krakau. 